\title{
Die Früherkennung zur Verbesserung der Lebensmittelsicherheit des Bundesamtes für Lebensmittelsicherheit und Veterinärwesen der Schweiz
}

\author{
Thomas Lüthi $^{1}$ [D . Isabelle Seger-Sauli ${ }^{1}$. Françoise Fridez ${ }^{1}$
}

Published online: 8 January 2022

(c) The Author(s) 2021

\section{Zusammenfassung}

Die Identifizierung von Gefahren in einem frühen Stadium ist ein zentraler Baustein der öffentlichen Gesundheit im Bereich der Lebensmittelsicherheit. Das Bundesamt für Lebensmittelsicherheit und Veterinärwesen der Schweiz verwendet seit 2018 ein System zur Früherkennung. Dieses stützt sich auf eine Gruppe von Fachpersonen, welche Informationen zu Gefahren identifizieren und periodisch bewerten. Der Beirat, ein wissenschaftliches Gremium von Experten und Expertinnen aus der Wirtschaft, von Hochschulen, Vollzugsbehörden und Referenzzentren erörtert anschließend die Themen und gibt seinerseits Empfehlungen an das Bundesamt für Lebensmittelsicherheit und Veterinärwesen ab. Insgesamt wurden bislang 37 Themen bearbeitet. Monatlich wird ein Newsletter zu Themen der Früherkennung publiziert. Zudem werden unregelmäßig bei Bedarf Briefing Letters mit einer vertieften Recherche zu einem Thema erstellt.

Schlüsselwörter Früherkennung $\cdot$ Schweiz $\cdot$ Lebensmittelsicherheit $\cdot$ Konzept $\cdot$ Umsetzung $\cdot$ Gefahren

\section{Early detection to improve food safety by the Federal Food Safety and Veterinary Office in Switzerland}

\begin{abstract}
The successful identification of hazards at an early stage is a cornerstone of public health in the area of food safety. The Swiss Federal Food Safety and Veterinary Office has been running an early detection system since 2018. A group of experts that identifies information concerning hazards and evaluates it periodically carries out the Federal Food Safety and Veterinary Office's early detection system. The advisory board, a scientific panel of experts from industry, universities, enforcement authorities and reference centres, discusses the topics and makes recommendations to the Swiss Federal Food Safety and Veterinary Office. To date, a total of 37 topics were handled. A monthly newsletter is published on early detection topics. In addition, "Briefing Letters" with an in-depth enquiry are issued on an irregular basis.
\end{abstract}

Keywords Early detection $\cdot$ Switzerland $\cdot$ Food safety $\cdot$ Concept $\cdot$ Field report $\cdot$ Hazards

Thomas Lüth

thomas.luethi@blv.admin.ch

$1 \quad$ Abteilung Risikobewertung, Fachbereich Toxikologie und Biologie, Gruppe Biologie und Früherkennung, Bundesamt für Lebensmittelsicherheit und Veterinärwesen, Schwarzenburgstrasse 155, 3003 Bern, Switzerland

\section{Einleitung}

Traditionell werden verschiedene Überwachungsansätze in der Lebensmittelsicherheit verwendet, um potenzielle Gefahren zu erkennen und zu bewerten und daraus Empfehlungen für mögliche Maßnahmen zu geben. Diese Ansätze sind wichtig, um unmittelbare Gefahren und Risiken zu erkennen. Es braucht daneben aber auch Prozesse, welche potenzielle Herausforderungen mittel- bis langfristige vorherzusagen vermögen, um wirksame Präventivmaßnahmen 
zu ermöglichen. Solche Ansätze werden seit vielen Jahren in verschiedenen Sektoren, allen voran in der Versicherungsbranche, angewandt und seit einiger Zeit nun auch in der Lebensmittelsicherheit zur Ermittlung potenzieller mittelund langfristiger Gefahren und Möglichkeiten eingesetzt (FAO 2014).

Die erfolgreiche Identifizierung von Gefahren in einem frühen Stadium ist von zentraler Bedeutung für die öffentliche Gesundheit im Bereich der Lebensmittelsicherheit. Viele Faktoren innerhalb und außerhalb der Lebensmittelproduktionssysteme können direkt und/oder indirekt das Auftreten von Gefahren und Risiken im Bereich der Lebensmittelsicherheit fördern.

Seit 2018 entwickelt und verwendet das Schweizer Bundesamt für Lebensmittelsicherheit und Veterinärwesen (BLV) ein Früherkennungssystem. Früherkennung beinhaltet dabei das Identifizieren, Sammeln, Verifizieren, Analysieren und Bewerten von Daten und Informationen. Innerhalb des Systems werden mögliche Gefahren und deren Risiken nach wissenschaftlichen Kriterien beurteilt. Die Früherkennung Sicherheit der Lebensmittelkette (FRESIL) erlaubt dem BLV, seine Aktivitäten und Ressourcen mittel- und langfristig auf die relevanten Bedrohungen auszurichten und adäquat mit ihnen umzugehen. Sie schafft die Voraussetzungen für ein rasches, effizientes und effektives Risikomanagement. In diesem Artikel wird die Konzeption der FRESIL des BLV sowie die damit verbundenen Erfahrungen dargelegt.

\section{Konzeption und Methodik}

Das BLV ist das Kompetenzzentrum des Bundes für die Lebensmittelsicherheit und Ernährung. Die Strategie legt fest, dass das BLV die Gesundheit von Mensch und Tier aktiv fördert und auf neue Aufgaben und Bedrohungen vorbereitet sein will (BLV 2021). Um dieses Ziel zu erreichen, räumt das Bundesamt der FRESIL einen hohen Stellenwert ein. Bei der Umsetzung der FRESIL werden Betrug und Täuschung, mikrobiologische, chemische sowie ernährungsbedingte Gefahren und Risiken berücksichtigt.

Die FRESIL hat sich bei der Erarbeitung der Konzeption an Früherkennungen anderer, ähnlicher Institutionen, wie beispielsweise dem Bayrischen Landesamt für Gesundheit und Lebensmittelsicherheit (LGL) und an Modellen aus der Versicherungswirtschaft orientiert. Die Konzeptionen der Schweizerischen Unfallversicherungsanstalt (SUVA) und des LGL dienten als Vorlage für die Früherkennung des BLV (Müller et al. 2016; SUVA 2013).

Die Früherkennung am BLV (Abb. 1) berücksichtigt Informationen aus verschiedenen Quellen. Sie stützt sich dabei auf eine Gruppe von Fachpersonen (genannt SEISMO), welche potenziell relevante Informationen identifizieren und ausgewählte Informationen miteinander besprechen. Einem Seismographen gleich, der Schwankungen der Erdkruste detektiert, sollen SEISMO Veränderungen im Bereich der Lebensmittelsicherheit aufspüren. Informationen können

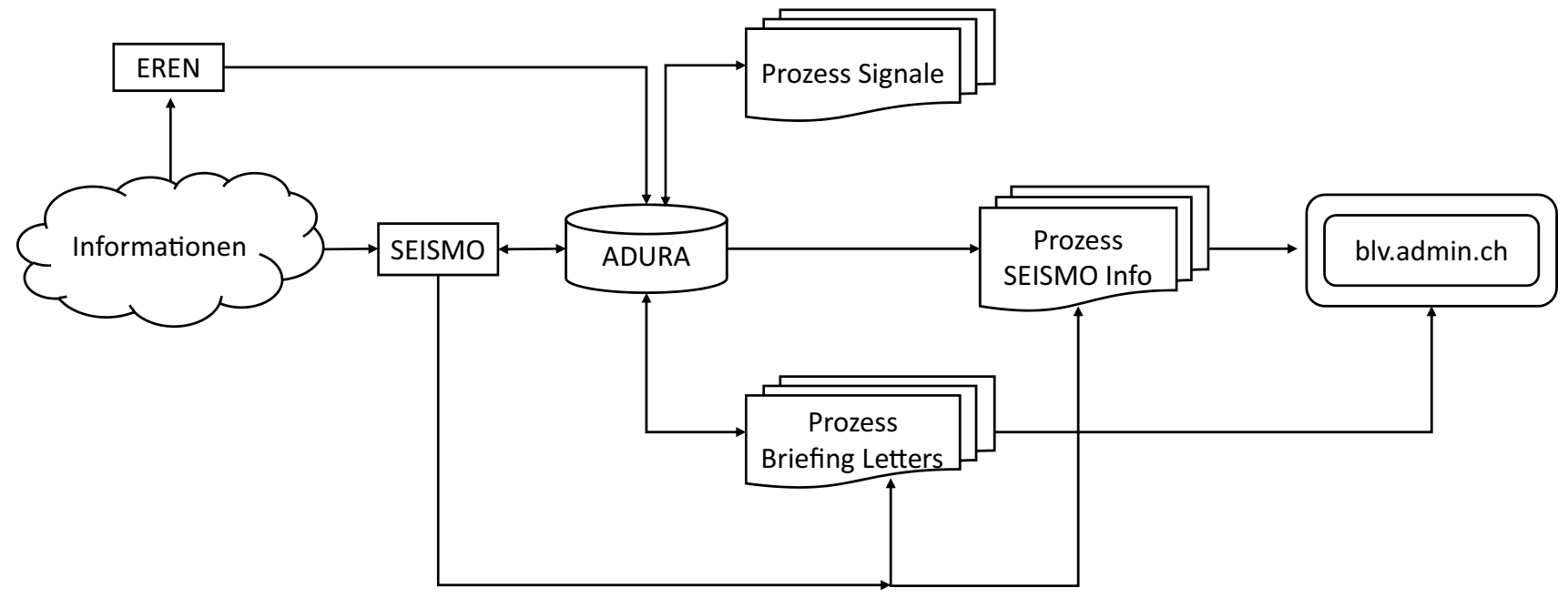

Abb. 1 Konzept der Früherkennung Sicherheit der Lebensmittel (FRESIL) des Bundesamtes für Lebensmittelsicherheit und Veterinärwesen (BLV). Dargestellt sind der Informationsfluss sowie die weiterführenden Prozesse und die daraus resultierenden Produkte: Signale, SEISMO Info und Briefing Letters. EREN: Emerging Risk Expert
Network der EFSA; SEISMO: Mitarbeitende der Früherkennung; ADURA: Datenbank der Früherkennung; SEISMO Info: monatlicher Newsletter; Briefing Letters: vertiefte Recherche zu einem Thema, auf der Webseite des BLV zugänglich 
aber auch von allen anderen Mitarbeitenden und weiteren interessierten Personen innerhalb und außerhalb des BLV an FRESIL übermittelt werden.

Das SEISMO-Netzwerk besteht aus rund 15 Personen, welche im BLV oder kantonalen Vollzugsbehörden tätig sind und diese Tätigkeit neben ihrer eigentlichen Arbeit wahrnehmen. Es beurteilt die Informationen bezüglich ihrer Wichtigkeit und Dringlichkeit und formuliert Empfehlungen. Der Beirat, ein wissenschaftliches Gremium von 12 Experten aus Wirtschaft, Hochschulen, Vollzugsbehörden und Referenzzentren diskutiert anschließend diese Themen und gibt seinerseits Empfehlungen für das BLV ab. Diese Empfehlungen werden dann durch Mitglieder der Geschäftsleitung geprüft und notwendige Aktivitäten ausgelöst.

Das BLV ist zudem Teil des internationalen Netzwerks „Emerging Risks Expert Network“ (EREN) der Europäischen Behörde für Lebensmittelsicherheit (EFSA), das regelmäßig Informationen über neue Gefahren austauscht, diese beurteilt und Maßnahmen diskutiert (EFSA 2020).

Informationen zu potenziellen Gefahren und Risiken werden systematisch durch die SEISMO in einer Datenbank, genannt ADURA ${ }^{1}$, erfasst. Diese kann von Fachpersonen von Bund und Kantonen, teilweise aber auch von der Öffentlichkeit und der Lebensmittelwirtschaft eingesehen werden. Als relevant eingestufte Informationen, welche im Früherkennungsprozess als solche identifiziert und beurteilt wurden, werden als Signale bezeichnet. Sie lösen im BLV Maßnahmen aus. Hierfür besteht ein Budget bei der Früherkennung, welches beispielsweise erlaubt rasch und unkompliziert vertiefende oder weitergehende Studien auszulösen. In einem monatlichen Newsletter, das „Seismo Info“", trägt das BLV die wichtigsten Informationen aus den Medien und wissenschaftlichen Publikationen zur Sicherheit von Lebensmitteln zusammen. Diese Informationen werden auch durch Briefing Letters kommuniziert. Informationen des ,SEISMO Info“ zu einem Sachverhalt gehen eher in die Breite, während sie in den Briefing Letters eher in die Tiefe gehen. Briefing Letters werden auf der Webseite des BLV der Lebensmittelwirtschaft, den Kontrollbehörden oder auch einem interessierten Publikum zur Verfügung gestellt.

\section{Erfahrungsbericht}

Das Konzept der Früherkennung wurde zu Beginn des Jahres 2019 am BLV offiziell umgesetzt. Bis Ende Juli 2021 sind rund 140 Informationen in Form von Meldungen von SEISMO, dem Beirat und weiteren Mitarbeitenden aus Bund und Kantonen eingegangen. Es wurden insgesamt 37 Signale (Tab. 1) bearbeitet, die zu weitergehenden Maßnahmen

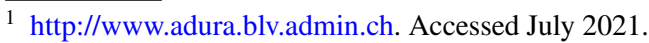

Tab. 1 Detektierte Signale der Früherkennung Sicherheit der Lebensmittel des Bundesamtes für Lebensmittelsicherheit und Veterinärwesen (BLV) von 2019-Juli 2021

\begin{tabular}{|c|c|c|}
\hline Nr. & Signal & Gefahr* \\
\hline 1 & Shigatoxin-produzierende Escherichia coli in Mehl & M \\
\hline 2 & $\beta$-Methylamino-L-alanin (BMAA) in Meeresfrüchten & $\mathrm{C}$ \\
\hline 3 & Azolresistenz von Aspergillus & $\mathrm{C}$ \\
\hline 4 & Organosilicon surfactant adjuvant & $\mathrm{C}$ \\
\hline 5 & Anthrachinon & $\mathrm{C}$ \\
\hline 6 & Mikroplastik & $\mathrm{C}$ \\
\hline 7 & Vibrio spp. in Meeresfrüchten & M \\
\hline 8 & Echinococcus spp. & M \\
\hline 9 & Mycobacterium avium ssp. paratuberculosis & M \\
\hline 10 & $\begin{array}{l}\text { Mikrobiologische Sicherheit Convenience Obst/ } \\
\text { Gemüse }\end{array}$ & M \\
\hline 11 & Hepatitis E & M \\
\hline 12 & Blockchain-Technologie in der Lebensmittelwirtschaft & - \\
\hline 13 & Anstieg enterohämorrhagische Escherichia coli & M \\
\hline 14 & Mykotoxine & $\mathrm{C}$ \\
\hline 15 & Cyclospora spp. & M \\
\hline 16 & E. coli $\mathrm{ST} 131$ & M \\
\hline 17 & $\begin{array}{l}\text { Tick-borne encephalitis virus (TBEV) in Milch- } \\
\text { produkten }\end{array}$ & M \\
\hline 18 & Nahrungsergänzungsmittel & $\mathrm{E}$ \\
\hline 19 & $\begin{array}{l}\text { Antimikrobielle Resistenzen bei pflanzlichen Lebens- } \\
\text { mitteln }\end{array}$ & $\mathrm{C}$ \\
\hline 20 & Organochloride beim Erhitzen von Sucralose & $\mathrm{C}$ \\
\hline 21 & Molkenpulver & - \\
\hline 22 & Bacillus cereus s.l. (thuringiensis) & M \\
\hline 23 & Cateringplattformen & M \\
\hline 24 & Fleischersatz & $\mathrm{C}$ \\
\hline 25 & Nanotechnologie & $\mathrm{C}$ \\
\hline 26 & Milchersatzprodukte & $\mathrm{C}$ \\
\hline 27 & Succinat Dehydrogenase Inhibitoren (SDHI) & $\mathrm{C}$ \\
\hline 28 & Arcobacter spp. & M \\
\hline 29 & Nacherntebehandlung Obst/Gemüse & M \\
\hline 30 & Streptococcus agalactiae ST283 in Fisch & M \\
\hline 31 & Salmonella Napoli & M \\
\hline 32 & Anisakis spp. & M \\
\hline 33 & Sojaöl & $\mathrm{E}$ \\
\hline 34 & Melatonin als Nacherntebehandlung & $\mathrm{C}$ \\
\hline 35 & Citrus Greening Disease & $\mathrm{C}$ \\
\hline 36 & Acinetobacter spp. & M \\
\hline 37 & Tierarzneimittel in Grundwasser & $\mathrm{C}$ \\
\hline
\end{tabular}

*Erklärung zu den Gefahrenarten: M: mikrobiologische, C: chemische und E: ernährungsbedingte Gefahren

führten. Davon betreffen 18 Signale mikrobiologische, 15 chemische und 2 ernährungsbedingte Gefahren. Zwei weitere Themen betrafen sogenannte Treiber, welche für die Entstehung von neuen Gefahren verantwortlich sein könnten. Unter Treibern werden Faktoren verstanden, welche das 
Vorkommen von Gefahren und Risiken der Lebensmittelsicherheit beeinflussen, wie dies beispielsweise beim Treiber Klimawandel diskutiert wird.

Der Newsletter „SEISMO Info“ wird monatlich in deutscher, französischer und in englischer Sprache erstellt und bis Ende Juli 2021 wurden 14 Briefing Letters in deutscher und teilweise auch französischer Sprache erarbeitet. Die Datenbank ADURA, welche möglicherweise für die FRESIL relevante Themen speichert, umfasst zurzeit mehr als 300 Einträge.

\subsection{Webbasierte Umfrage zur FRESIL des BLV}

Im Juni 2021 wurde unter den Nutzern der FRESIL, dem Beirat sowie den SEISMO eine webbasierte Umfrage durchgeführt. Ziel war es, die Bedürfnisse und Erwartungen der verschiedenen Gruppen an die Früherkennung kennen zu lernen und basierend darauf FRESIL weiterzuentwickeln. Dazu wurden allgemeine Fragen zur Früherkennung und den daraus resultierenden Produkten, wie zum Beispiel zum Newsletter SEISMO Info, zu Briefing Letters, der Webseite der Früherkennung und der Datenbank ADURA gestellt. Ein Workshop mit Mitgliedern aus SEISMO und Beirat ergänzte die Erkenntnisse der Umfrage.

Insgesamt haben 62 Personen die Umfrage ${ }^{2}$ vollständig beantwortet. Rund 22\% der Antwortenden waren entweder SEISMO oder Beiratsmitglieder. Rund $67 \%$ bezeichneten sich primär als Leser des Newsletters „SEISMO Info“. Die Nutzer der Früherkennung des BLV arbeiten in der Lebensmittelwirtschaft (33\%), in Lebensmittelkontrollbehörden (22\%), in Bundesbehörden (17\%), an Hochschulen (5\%) und weiteren Organisationen, wie Analyselaboratorien, in Beratungsfirmen, oder Branchenorganisationen (insgesamt $17 \%)$.

Die Antwortenden der Umfrage gaben an, dass das BLV ihre Erwartungen an die FRESIL vollständig (40\%) oder zu großen Teilen (40\%) erfülle. Als wichtigstes Element der Früherkennung des BLV hat sich der Newsletter ,SEISMO Info" entpuppt; $85 \%$ bezeichneten diesen als nützlich. Die Webseite der Früherkennung wurde von $81 \%$, die Briefing Letters von 68\% und die Datenbank ADURA von 47\% der Befragten als nützlich bezeichnet, wobei bei letzteren der Anteil der Personen, welche keine Bewertung abgaben, bei $25 \%$ (Briefing Letters) und 44\% (ADURA) lag.

\footnotetext{
${ }^{2}$ Bericht: Umfrage zur Früherkennung für die Sicherheit der Lebensmittel 2021 (Webseite des BLV https://www.blv.admin.ch/dam/blv/ de/dokumente/lebensmittel-und-ernaehrung/publikationen-forschung/ umfrage-frueherkennung-2021.pdf.) Accessed November 2021.
}

\subsection{Stärken der FRESIL}

Die vorgestellte Konzeption hat sich grundsätzlich bewährt und ist im BLV etabliert. Der Früherkennungsprozess berücksichtigt die unterschiedlichsten Themen. Das SEISMO-Gremium und der Beirat sind motiviert, rund drei- bis viermal jährlich Themen zu diskutieren und zu entscheiden, wie relevant diese für das BLV sein könnten. Dieser fachliche Austausch wird von den SEISMO und dem Beirat sehr geschätzt. Er ermöglicht es der Schweiz zudem, sich aktiv in das Netzwerk „EREN“ einzubringen, um neue Themen zu setzen oder um Ergebnisse als Follow-up zu bereits diskutierten Signalen zu präsentieren. Umgekehrt können Themen des EREN in die nationale Früherkennung eingebracht und unter den nationalen Gegebenheiten diskutiert werden. Das „SEISMO Info“ ist aktuell das zentrale Kommunikationsmittel um die Belange der Früherkennung zu kommunizieren. Rund 92\% der Nutzer des „SEISMO Info" sind mit diesem zufrieden.

Mit einem Ressourcenaufwand von rund 1,2 Stellen (Prozesseigner [BLV] und Mitarbeitende), sowie einem Aufwand für die SEISMO von rund 25 Stunden pro Person und Jahr, respektive rund 20 Stunden pro Person und Jahr für den Beirat, wird eine Vielzahl von Informationen und Signalen durch die Früherkennung bereitgestellt.

\subsection{Schwächen der FRESIL}

Die FRESIL des BLV stützt sich - konzeptbedingt und bedingt durch die limitierten Ressourcen - auf wenige Personen. Dies ist einerseits eine Stärke, andererseits aber auch ein Schwachpunkt, da dadurch die Früherkennung des BLV stark personenabhängig ist. Dies gilt für die SEISMO, den Beirat und den Prozesseigner.

Wie bei vielen ähnlich gelagerten Projekten nimmt nach anfänglich hoher Motivation, diese gemessen an der Zahl der Informationen an die Früherkennung sukzessive ab (2019:99; 2020:32; 2021 Ende Juli: 8).

Bemängelt wurde von den SEISMO und den Beiratsmitgliedern die mangelnde Transparenz bei der Auswahl der zu diskutierenden Themen. Eine weitere Schwierigkeit besteht zudem darin, dass für potenzielle Signale naturgemäß nur unzureichende Daten zur Verfügung stehen, um eine Beurteilung der Relevanz vornehmen zu können.

Erkannte Signale sind in der Regel nicht im Tagesgeschäft zu bearbeiten, sie verlangen eine Projektorganisation. Da Daten oftmals fehlen, sind zudem meist weitergehende Literaturstudien in Auftrag zu geben oder anhand von Prävalenzstudien beispielsweise das Vorkommen eines bestimmten Erregers zu verifizieren. Solche Projekte sind zu planen, zu budgetieren und anschließend, oft extern, umzusetzen. 
Liegen die verschiedenen Informationen und eine finale Beurteilung vor, dann erfolgt die Übergabe des Themas an das Risikomanagement. Gegebenenfalls kollidieren Themen der Früherkennung mit solchen des Tagesgeschäftes, welche ebenfalls als prioritär eingestuft wurden.

Die Frage nach der erzielten Wirkung der Früherkennung kann oft nur qualitativ - und dann auch nur zum Teil - beantwortet werden. Die Zeitspanne seit der Etablierung der Früherkennung ist dafür zu kurz.

\section{Fazit}

Die FRESIL konnte am BLV problemlos eingeführt und umgesetzt werden. Sie hat sich grundsätzlich bewährt. Die Entscheidung, sich primär auf Personen zu stützen, war richtig, ist nun aber an einem Punkt angelangt, an welchem über eine Integration von künstlicher Intelligenz zur Identifikation und Bewertung potenziell relevanter Themen nachzudenken ist. Die größte Herausforderung jeder Früherkennung ist es, aus der Vielzahl der Informationen diejenigen Informationen herauszufiltern, welche für die Sicherheit der Lebensmittel zukünftig auch tatsächlich relevant sind. Ein hybrider Ansatz, der Mensch und künstliche Intelligenz (Algorithmen) verknüpft, ist zu prüfen.

Danksagungen Die Autoren bedanken sich bei den SEISMO und dem Beirat für ihre wertvolle Arbeit; der Dank geht auch an die Teilnehmenden der Umfrage zur Früherkennung sowie an Frau Dr. B. Müller vom Bayrischen Landesamt für Gesundheit und Lebensmittelsicherheit (LGL) für die kritische Durchsicht des Manuskriptes und ihre wertvollen Hinweise.

Funding Not applicable.

Availability of data and material Not applicable.

Code availability (software application or custom code) Not applicable.

\section{Declarations}

Conflict of interest Die Autoren erklären, dass sie sich in keinem Interessenskonflikt befinden.
Open Access This article is licensed under a Creative Commons Attribution 4.0 International License, which permits use, sharing, adaptation, distribution and reproduction in any medium or format, as long as you give appropriate credit to the original author(s) and the source, provide a link to the Creative Commons licence, and indicate if changes were made. The images or other third party material in this article are included in the article's Creative Commons licence, unless indicated otherwise in a credit line to the material. If material is not included in the article's Creative Commons licence and your intended use is not permitted by statutory regulation or exceeds the permitted use, you will need to obtain permission directly from the copyright holder. To view a copy of this licence, visit http://creativecommons.org/licenses/by/4.0/.

\section{Literatur}

BLV (Bundesamt für Lebensmittelsicherheit und Veterinärwesen) (2021) Strategien. https://www.blv.admin.ch/blv/de/home/dasblv/strategien.html. Accessed 30 Sept 2021

EFSA (European Food Safety Authority), Afonso A, Garcia Matas R, Gkrintzali G, Maggiore A, Merten C, Rortais A, Robinson T (2020) Technical report on EFSA's activities on emerging risks in 2019. EFSA supporting publication 2020:EN-1924. https://doi. org/10.2903/sp.efsa.2020.EN-1924. Accessed 30 Sept 2021

FAO (Food and Agriculture Organisation of the United Nations) (2014) Horizon Scanning and Foresight. An overview of approaches and possible applications in Food Safety. FAO Early Warning/Rapid Alert and Horizon Scanning, Food Safety Technical Workshop Rome, 22-25 October 2013. http://www.fao.org/3/i4061e/i4061e. pdf. Accessed 30 Sept 2021

Müller B, Verhaelen K, Eberlein V, Celik BÜ, Butzenlechner M, Busch U, Weidner C, Holtmannspötter GW, Wallner P (2016) Etablierung eines Frühwarnsystems zur Erkennung lebensmittelbedingter Risiken in Bayern - risikoorientierte Lebensmittelüberwachung weiter gefasst. J Verbr Lebensm 11:9-18. https://doi.org/10.1007/ s00003-015-0978-0

SUVA (Schweizerische Unfallversicherungsanstalt), Roos GT, Biland S (2013) Früherkennungsradar Zukünftige Chancen und Risiken für die Prävention von Unfällen und Berufskrankheiten, Bestellnummer 2965.d 2. Auflage, Mai 2013. https://www.suva.ch/de$\mathrm{CH} /$ material/Dokumentationen/frueherkennungsradar\#sch-froms earch\%23. Accessed 30 Sept 2021

Publisher's Note Springer Nature remains neutral with regard to jurisdictional claims in published maps and institutional affiliations. 